\title{
Evaluation of Effect of Music on Human Nervous System by Heart Rate Variability Analysis Using ECG Sensor
}

\author{
Min-Hao Wu and Ting-Cheng Chang* \\ College of Information Engineering, Guangzhou Panyu Polytechnic, Guangdong 511483, China
}

(Received July 20, 2020; accepted January 25, 2021)

Keywords: ECG, music preference, heart rate variability, physiological signals, emotional heart rate values

To investigate the effect of music on emotion, the physiological responses to music were analyzed with respect to heart rate variability (HRV) using an electrocardiogram (ECG) sensor. For the experiment, music was categorized by its bits per minute (BPM) and played while monitoring the HRV of the participants. The obtained data were analyzed to obtain parameters in the frequency domain analysis. The indicators of the autonomic nervous system (ANS) changed with the BPM of music and represented the emotional state of the participants. In particular, total power (TP), high- and low-frequency powers, and high- and low-frequency power ratios (HFR and LFR, respectively) changed when listening to fast (120-140 BPM), intermediate (60-80 BPM), and slow (less than 40 BPM) music. Fast music enhanced the autonomic sympathetic nervous system, thus increasing LFR and TP, but HFR decreased owing to the reduced activity of the parasympathetic nerve. Intermediate music inhibited the activity of the autonomic sympathetic nerve, leading to decreased LFR and increased HRF owing to the enhanced activity of the parasympathetic nerve. Slow music did not change the activity of the autonomic sympathetic nerve. These results suggest that music can be used to lessen or prevent stress and enhance work performance.

\section{Introduction}

The autonomic nervous system (ANS) controls the functions of the human heart muscle and glands for regulating the heartbeat, breathing, blood pressure, digestion, and metabolism. ${ }^{(1)}$ In a normal circumstance, the sympathetic and parasympathetic nerves of the ANS are balanced to maintain homeostasis in a human body. However, an ANS imbalance due to stress accelerates the heart rate by the secretion of hormones and causes, for example, sleep disorder, stomach ailments, headaches, muscle tension, and tinnitus. These symptoms are significantly related to cardiovascular diseases. ${ }^{(2,3)}$ Indicators of the ANS include skin reflex tests, sweating responses, and biochemical tests. These are usually measured by invasive methods that require expensive instruments and cause pain to the patients. In contrast, heart rate variability (HRV) is a measure of the ANS that can be determined noninvasively. When the parasympathetic nervous system is

*Corresponding author: e-mail: 18250922163@163.com

https://doi.org/10.18494/SAM.2021.3040 
dominant, the heartbeat slows to increase HRV. ${ }^{(4)} \mathrm{HRV}$ is obtained from an electrocardiogram (ECG) and measured as the R-R interval, which is defined as the time between heartbeats and is calculated in the time and frequency domains. HRV is related to the level of stress that is known to cause an autonomic dysfunction and the impairment of the body system, which are major causes of cardiovascular diseases, the leading cause of death in the world for many years. ${ }^{(5,6)}$

There have been many efforts to find ways to lessen stress through various activities. Music has long been considered to reduce physiological and psychological stress. The effects of music that have been suggested include good sleep quality, relaxation, relief of anxiety, and reduction of pain. Davidson et al. found that music changed the heart rate of a hypnotized person. ${ }^{(7)}$ Hiraga et al. observed visual changes of the human face caused by the emotional response to a musical performance. ${ }^{(8)}$ Tsihrintzis et al. found that a keyboard-stroke pattern's rhythm (tempo) affects emotional facial expressions as a physiological response such as excitement and relaxation. ${ }^{(9)}$ Many results of HRV studies have suggested that music reduces the parasympathetic nerve activity even though individuals have different music preferences.

Most traditional ECG monitoring can only be performed in hospitals. Timely monitoring and diagnosis cannot be performed on people at home. Moreover, it is difficult for people to have an intuitive understanding of their ECG and emotional conditions, and they cannot know their emotional health status at a given time. An HRV instrument can monitor users. An emotion sensor can detect heart health, but more importantly, it can reflect the emotional fluctuations of the wearer. If the wearer is in a state of excitement or depression for a long time, he or she may have a specific mental illness. Many people have psychological problems but are unaware of them, and an emotion sensor can promptly address this problem, inform the wearer of their mental health, and allow timely adjustment to avoid the accumulation of mental health problems such as depression. Preventing such problems before they occur will give the wearer greater peace of mind.

In this study, we aim to investigate how music changes the human emotion and to prove that stress is reduced by listening to music in experiments with HRV measurement. ${ }^{(10,11)}$ In the experiment, we used a miniaturized ECG with appropriate sensors to monitor and record HRV, and then determined whether there are changes in emotion by processing the HRV data. The results of this study may provide information on how to reduce stress.

\section{Methods}

\subsection{Measurement system}

The system used for measuring HRV is shown in Fig. 1. Participants of the experiment wore a BeneGear ECG belt on their chest. The heartbeat was monitored using the sensors embedded in the conductive rubber of the belt. The sensors detected and amplified the potential differences generated by the heartbeat. Then the potential differences were converted to electric signals and transmitted to a receiving device (a mobile device or a computer) through a Bluetooth 4.0 module. Then, a program in the receiving device processed the heartbeat rate (HR) and R-R interval to calculate the parameters and indicators for use in frequency domain analysis. 


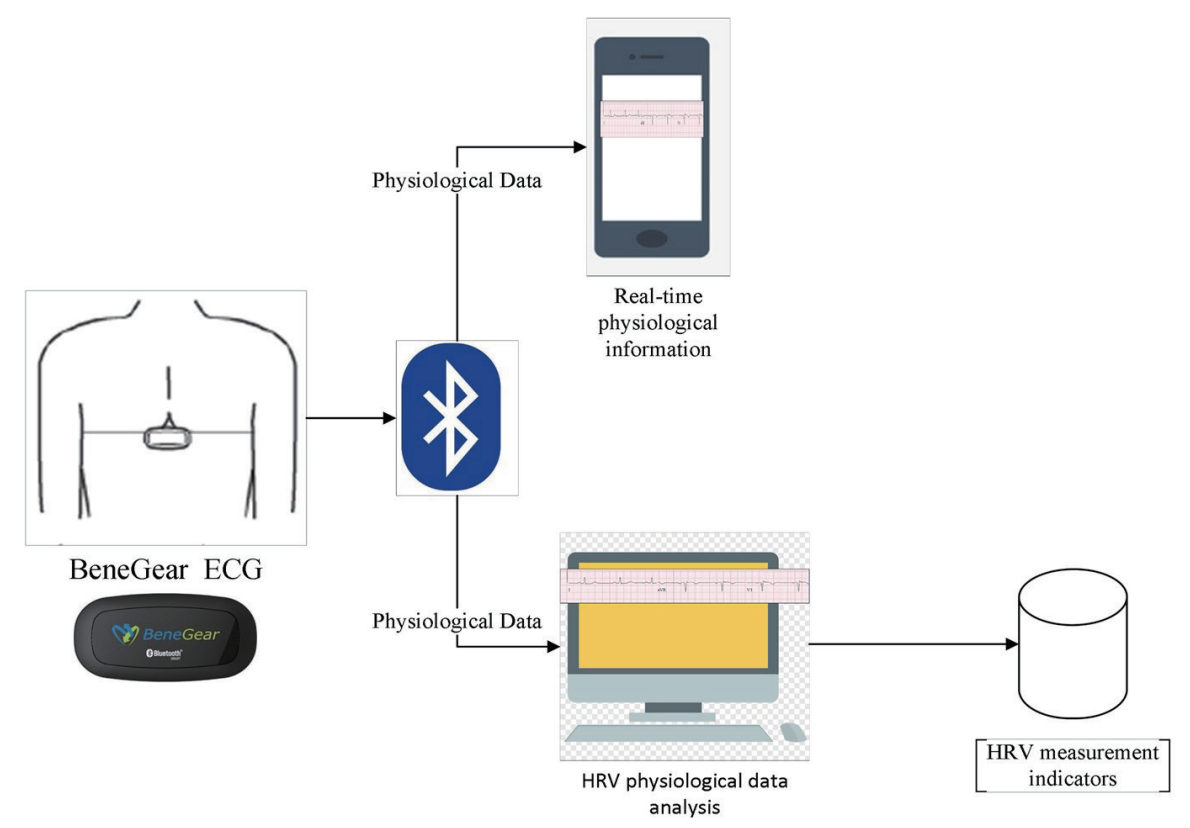

Fig. 1. (Color online) Schematic diagram of the HRV measurement system.

\subsection{Participants}

Fourteen male students aged 18-22 years participated in the experiment. All participants were selected in accordance with the following criteria.

(1) Exercised regularly

(2) Capable of oral communication

(3) No significant diseases, especially cardiovascular diseases

(4) No need to take regular medication

Before the experiment, personal information (i.e., date of birth, height, weight, and stress) was collected.

\subsection{Experiment}

The experiment was conducted once a week (for eight weeks) for each participant to measure the activities of sympathetic and parasympathetic nerves without music and with listening to music of different rhythms such as less than 40 bits per minute (BPM, slow music), 60-80 BPM (intermediate music), and 120-140 BPM (fast music). Each participant had eight sessions in the experiment. In one session, the participant rested for $15 \mathrm{~min}$ before the experiment to become comfortable in the test environment. One session lasted $30 \mathrm{~min}$ with three parts. In each $10 \mathrm{~min}$ part, the participants relaxed silently for $5 \mathrm{~min}$ and then listened to music for the following 5 min. This was performed with fast, intermediate, and slow music. During the session, the HRVs of the participants were monitored and recorded for $30 \mathrm{~min}$. External disturbances were shut out in and around the experiment room during the experiment. The scheme of the experiment is shown in Fig. 2. 


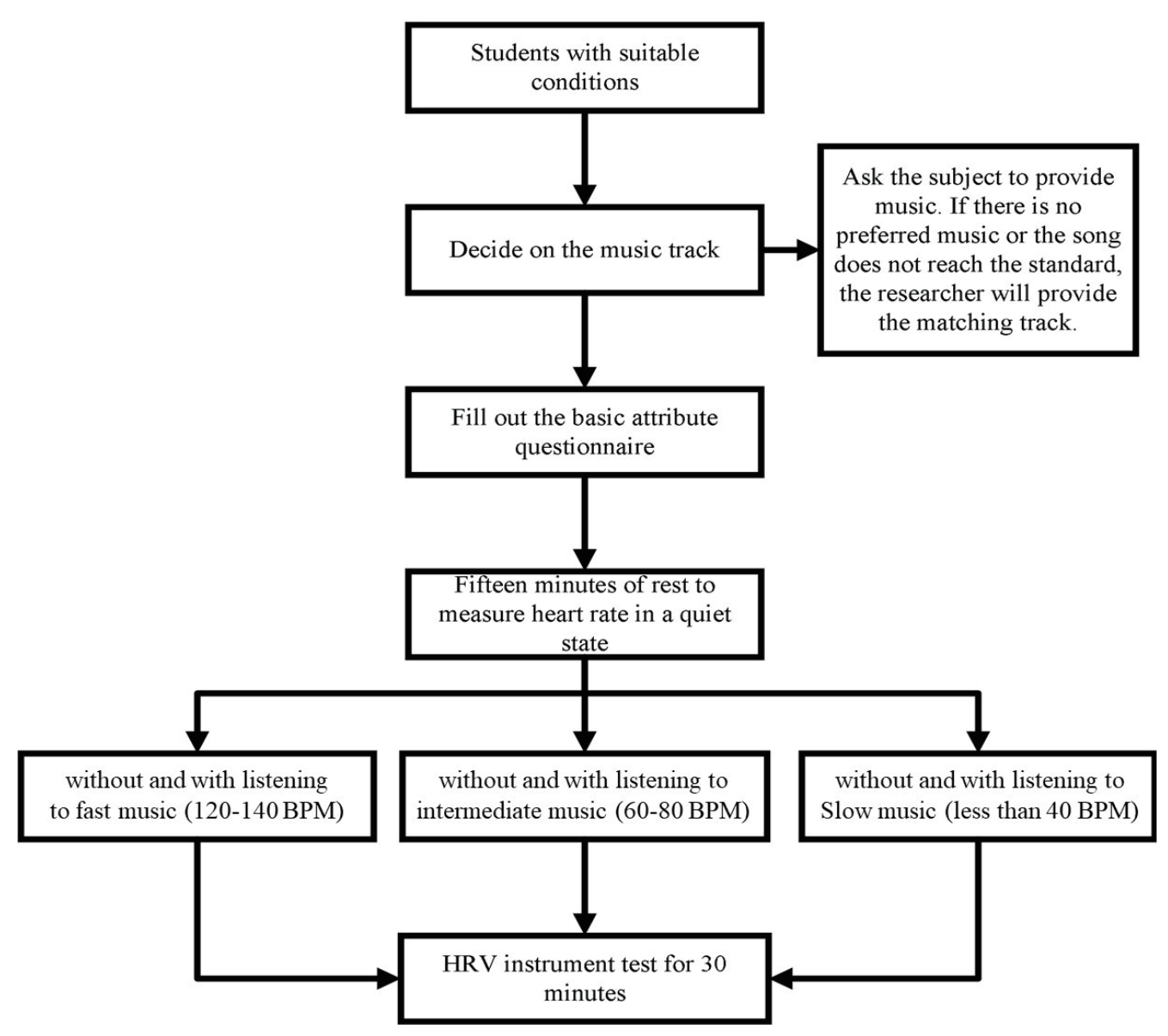

Fig. 2. Scheme of the experiment in this study.

\subsection{HRV analysis}

Acharya et al. proposed a method of determining the health status and physiological adjustment of the human body on the basis of the change in heart rate. ${ }^{(12)}$ As the human heart does not beat regularly at all times, the interval between heartbeats varies continuously. The change in heartbeat rate enables the medical diagnosis of cardiovascular diseases, neurological diseases, and diabetes. The analysis process for HRV is shown in Fig. 3.

\section{Time domain analysis of $H R V$}

The R-R interval is a significant parameter and necessary data in the time domain analysis of HRV. Table 1 shows the common parameters of the time domain analysis of HRV.

\section{Frequency domain analysis of $H R V$}

Among the frequency domain methods for analyzing HRV, the most commonly used calculation method is the fast Fourier transform (FFT), which employs the power spectral density (PSD). ${ }^{(16,17)}$ In frequency domain analysis, indicators are defined on the basis of the 


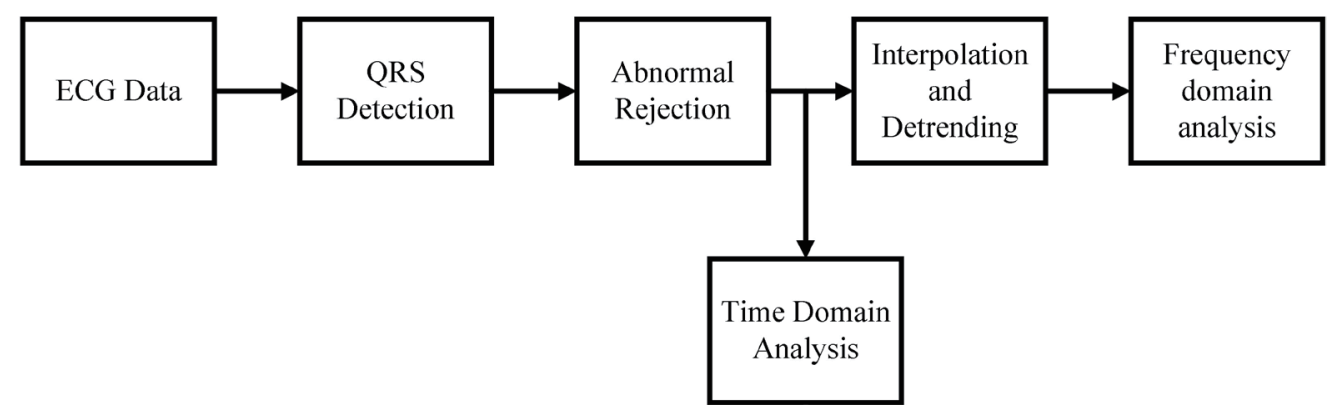

Fig. 3. Analysis process for HRV.

Table 1

Commonly used time domain analysis parameters for detecting HRV. ${ }^{(13-15)}$

\begin{tabular}{lrl}
\hline Indicator & Unit & \multicolumn{1}{c}{ Description } \\
\hline $\begin{array}{l}\text { Standard deviation of all normal-to-normal } \\
\text { intervals (SDNN) }\end{array}$ & $\mathrm{ms}$ & $\begin{array}{l}\text { The standard deviation of a normal sinus interval is determined } \\
\text { by calculating the short-term average normal heartbeat } \\
\text { interval, usually } 5 \text { min, and then calculating the average } \\
\text { standard deviation throughout the process. }\end{array}$ \\
\hline $\begin{array}{l}\text { Standard deviation of average } \\
\text { normal-to-normal interval index (SDANN) }\end{array}$ & $\mathrm{ms} \quad \begin{array}{l}\text { Calculate the short-term average normal heartbeat interval } \\
\text { (about } 5 \text { min) and then the average standard deviation of the } \\
\text { whole process. }\end{array}$ \\
$\begin{array}{l}\text { Square root of the mean of the sum of the } \\
\text { squares of differences between adjacent } \\
\text { NN intervals (R-MSSD) }\end{array}$ & $\mathrm{ms}$ & $\begin{array}{l}\text { Root mean square of the sum of squares of the difference } \\
\text { between normal heartbeat intervals. }\end{array}$ \\
NN50 & $\%$ & $\begin{array}{l}\text { Number of cases where the difference between normal } \\
\text { heartbeat intervals exceeds 50 ms. }\end{array}$ \\
\hline PNN50 & None & $\begin{array}{l}\text { Divide the number of intervals between all pairs of adjacent } \\
\text { normal heartbeats in the ECG with a difference of more than } \\
50 \text { ms by the total number of normal heartbeat intervals in the } \\
\text { measured ECG. }\end{array}$ \\
\hline
\end{tabular}

frequency ranges shown in Table 2. The frequency domain analysis of HRV can show whether a patient has heart problems, and HRV is one of the essential physiological parameters closely related to emotions. The calculated normalized low-frequency power ratio (normalized LF, $\mathrm{nLF}$ ) represents the degree of sympathetic activation in the autonomic nervous system. Lowfrequency power, normalized low-frequency power ratio, high-frequency power, and normalized high-frequency power can represent the degree of balance and activation of the sympathetic and parasympathetic nervous systems in the calculation of the normalized low-frequency power ratio and normalized high-frequency power ratio. Removing the frequency domain power other than the high- and low-frequency powers can prevent the balance between the sympathetic and parasympathetic nervous systems from changing. The total power also changes, and the balance of the autonomic nervous system cannot be accurately displayed. The activity of the sympathetic and parasympathetic nervous systems of the autonomic nervous system has a significant correlation with emotions: the greater the activity of the sympathetic nervous system, the more the nervous, ease, and burnout state. The greater the parasympathetic nervous system's activity, the more joyful and the calmer the mood, and the more likely the person is to 
Table 2

Frequency ranges of indicators defined by frequent domain analysis of HRV. ${ }^{(16)}$

\begin{tabular}{lcc}
\hline Indicator & Frequency $(\mathrm{Hz})$ & \multicolumn{1}{c}{ Measurement } \\
\hline Total power (TP) & $0-0.4$ & Variation in normal heartbeat interval \\
\hline $\begin{array}{l}\text { Ultralow-frequency power } \\
\text { (ULFP) }\end{array}$ & $0-0.003$ & $\begin{array}{c}\text { Variation during normal heartbeat } \\
\text { in ultralow-frequency range }\end{array}$ \\
\hline $\begin{array}{l}\text { Very low frequency power } \\
\text { (VLFP) }\end{array}$ & $0.003-0.04$ & $\begin{array}{l}\text { Variation during normal heartbeat } \\
\text { in extremely low frequency range }\end{array}$ \\
$\begin{array}{l}\text { Low-frequency power } \\
\text { (LFP) }\end{array}$ & $0.003-0.04$ & $\begin{array}{c}\text { Sympathetic nerve activity } \\
\text { Normalized low-frequency } \\
\text { power (nLFP) }\end{array}$ \\
$\begin{array}{l}\text { High-frequency power } \\
\text { (HFP) }\end{array}$ & \begin{tabular}{l} 
Degree of sympathetic and parasympathetic balance and activation \\
\hline $\begin{array}{l}\text { Normalized high-frequency } \\
\text { power (nHFP) }\end{array}$
\end{tabular} & $\begin{array}{c}\text { Degree of sympathetic nerve activation } \\
\text { Degree of sympathetic and parasympathetic balance and activation }\end{array}$ \\
\hline
\end{tabular}

be experiencing fatigue. It can be seen that when the proportion of low-frequency components is higher, the emotions are in a state of excitement, tension, and anxiety, and when the proportion of high-frequency components is higher, the emotions are in a state of calm, relaxation, and fatigue.

Indicators of the parasympathetic nerve activity and autonomic nerve regulation change with work pressure, emotion, and physical activity. Research studies on the ANS revealed that a reduction in regulatory ability owing to enhanced sympathetic nerve and decreased parasympathetic activities increased the risk of cardiovascular diseases. ${ }^{(18)}$ Regulation of the ANS is possible through the administration of drugs, mood change, and exercise. Previous studies revealed that listening to music may trigger physical and psychological reactions. Thus, music therapy is a common treatment for physical and psychological diseases as music stimulates the limbic system of the midbrain and secretes neurotransmitters such as dopamine. Several groups have pointed out that listening to intermediate music during exercise contributes to heartbeat regulation by increasing the parasympathetic nerve activity, which inhibits the increase in HR.

In the frequency domain analysis of HRV, the important physiological indicators related to emotion are defined as follows.

$$
\text { Total power }(T P)=H F+L F+V L F
$$

Very low frequency power $(V L F P)=\exp (V L F)$

$$
\text { Low-frequency power }(L F P)=\exp (L F)
$$

Normalized low-frequency power $(n L F P)=L F /(T P-E L F P)$

(ELFP: Extremely low frequency power)

$$
\text { High-frequency power }(H F P)=\exp (H F)
$$


Normalized high-frequency power $(n H F P)=H F /(T P-E L F P)$

$$
\text { Frequency ratio }(F R)=L F / H F
$$

$$
\begin{aligned}
& \text { High-frequency power ratio }(\mathrm{HFR})=H F /(T P-V L F) \\
& \text { Low-frequency power ratio }(L F R)=L F /(T P-V L F)
\end{aligned}
$$

The various indicators of the HRV frequency domain and the corresponding emotional characteristics are described in Table 3.

In this study, the indicators of frequency domain analysis were obtained and analyzed by statistical methods such as independent $t$-tests with the two-sample equal-variance hypothesis, descriptive statistics, and the paired sample $t$-test.

Table 3

HRV frequency domain indicators and emotional state. ${ }^{(22)}$

\begin{tabular}{lcccc}
\hline Indicator & Unit & Frequency $(\mathrm{Hz})$ & Autonomic nerve activity & Emotional characteristics \\
\hline ULFP & $\mathrm{ms}^{2}$ & $0-0.003$ & - & Undefined \\
\hline VLFP & $\mathrm{ms}^{2}$ & $0.003-0.04$ & - & Undefined \\
\hline LFP & $\mathrm{ms}^{2}$ & $0.04-0.15$ & Sympathetic nerve & Nervousness, excitement, and anxiety \\
\hline HFP & $\mathrm{ms}^{2}$ & $0.15-0.4$ & Parasympathetic nerve & Pleasure, calmness, relaxation, and burnout \\
\hline nLFP & None & - & Sympathetic nerve & Nervousness, excitement, and anxiety \\
\hline nHFP & None & - & Parasympathetic nerve & Pleasure, calmness, relaxation, and burnout \\
\hline
\end{tabular}

\section{Results and Discussion}

\subsection{Indicators of ANS}

\section{Fast music}

Table 4 shows the measured frequency domain indicators of the participants without and with listening to fast music with 120-140 BPM. LFR was determined to be $45-53 \%$ and $48-$ $57 \%$ without and with fast music, respectively. The results of the paired sample $t$-test showed a significant effect of fast music on LFR $(F=0.00, p<0.05)$ (Fig. 4). This means that the sympathetic nerve activity was reduced via an inhibitory effect. HFR was determined to be $35-$ $40 \%$ and $37-45 \%$ without and with listening to fast music, respectively. The result of the paired sample $t$-test showed the significance of the effect of fast music on HFR $(F=0.00, p<0.05)$ (Fig. $5)$. This showed that fast music stimulated the parasympathetic nerve activity and endowed the participants with a relaxing emotion. This implies that the participants had a certain degree of stress that was relieved by listening to fast music. 
Table 4

Frequency domain indicators measured without and with fast music.

\begin{tabular}{|c|c|c|c|c|c|c|c|}
\hline \multicolumn{8}{|c|}{ Without fast music } \\
\hline Experiment Session & LFP & HFP & $\mathrm{FR}(\mathrm{LF} / \mathrm{HF})$ & VLFP & LFR (\%) & HFR (\%) & TP \\
\hline 1 & 498.55 & 435.58 & 2.44 & 517.73 & 50 & 38 & 1451.86 \\
\hline 2 & 290.05 & 292.22 & 1.69 & 638.04 & 50 & 40 & 1127.93 \\
\hline 3 & 420.06 & 404.01 & 2.28 & 430.16 & 54 & 35 & 1254.24 \\
\hline 4 & 480.99 & 161.53 & 1.65 & 407.23 & 53 & 38 & 1778.35 \\
\hline 5 & 260.03 & 183.34 & 1.96 & 747.99 & 57 & 34 & 1771.09 \\
\hline 6 & 416.68 & 332.2 & 2.1 & 627.27 & 48 & 35 & 1510.3 \\
\hline 7 & 500.25 & 324.77 & 2.29 & 618.71 & 55 & 35 & 1443.73 \\
\hline 8 & 350.42 & 335.66 & 2.42 & 795.83 & 55 & 35 & 2406.24 \\
\hline \multicolumn{8}{|c|}{ With fast music } \\
\hline Experiment Session & LFP & HFP & FR(LF/HF) & VLFP & LFR (\%) & HFR (\%) & TP \\
\hline 1 & 477.70 & 591.68 & 1.57 & 904.37 & 46 & 43 & 1973.74 \\
\hline 2 & 668.05 & 532.84 & 1.73 & 552.52 & 48 & 39 & 2501.59 \\
\hline 3 & 413.52 & 502.07 & 1.23 & 728.02 & 45 & 45 & 1643.61 \\
\hline 4 & 631.16 & 543.39 & 1.40 & 695.42 & 46 & 43 & 1630.19 \\
\hline 5 & 284.79 & 285.06 & 1.83 & 791.06 & 53 & 37 & 1216.13 \\
\hline 6 & 343.83 & 374.87 & 1.37 & 675.26 & 47 & 42 & 1393.95 \\
\hline 7 & 462.60 & 468.94 & 1.43 & 1152.38 & 49 & 41 & 1811.34 \\
\hline 8 & 490.30 & 498.95 & 1.69 & 779.52 & 49 & 41 & 1768.77 \\
\hline
\end{tabular}

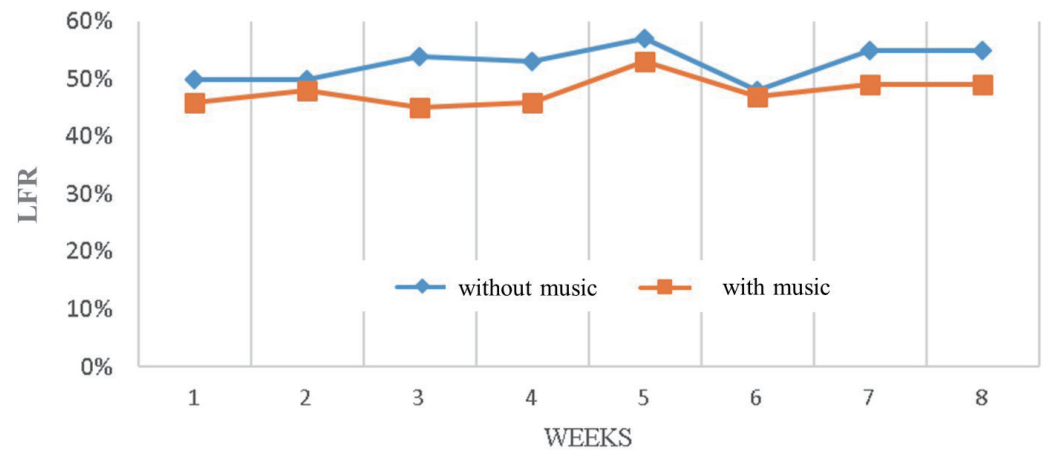

Fig. 4. (Color online) Changes in LFR without and with fast music in the experiment period.

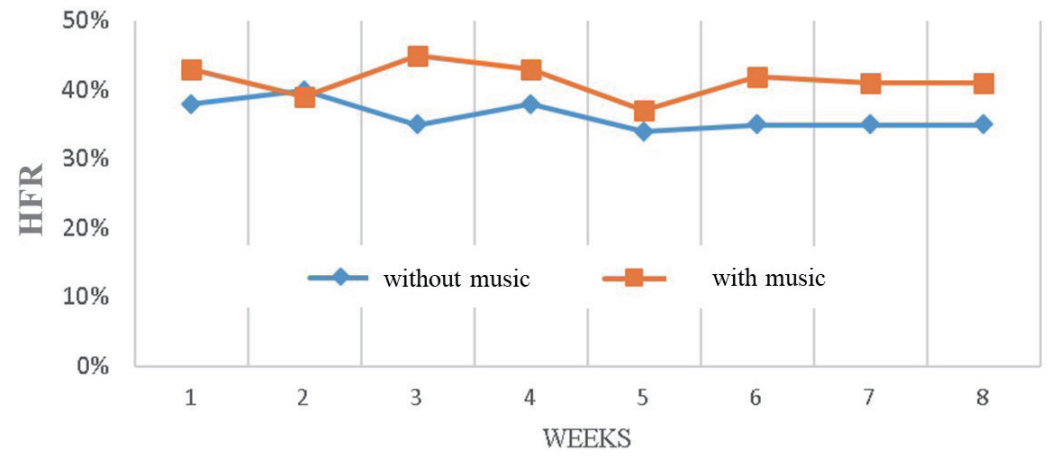

Fig. 5. (Color online) Changes in HFR without and with fast music in the experiment period. 


\section{Intermediate music}

Table 5 shows the changes in indicators when listening to intermediate music. A gradual increase in total power (TP) was observed and the difference between TPs with and without intermediate music became larger as the experiment was repeated. This result suggests that intermediate music increased the ANS activity more as the participants became accustomed to listening to intermediate music. LFR increased significantly with intermediate music according to the paired $t$-test result $(F=0.019, p<0.05)$ (Fig. 6). This suggests an increase in sympathetic nerve activity when listening to intermediate music, resulting in the excitement effect. HFR in the case with intermediate music was significantly lower than that without intermediate music $(F=0.010, p<0.05)$ (Fig. 7). Listening to intermediate music caused a decrease in the parasympathetic nerve activity and an increase in the sympathetic nerve activity, which boosted the spirit.

Table 5

Indicators measured without and with intermediate music.

\begin{tabular}{|c|c|c|c|c|c|c|c|}
\hline \multicolumn{8}{|c|}{ Without intermediate music } \\
\hline Experiment Session & LFP & HFP & FR (LF/HF) & VLFP & $\operatorname{LFR}(\%)$ & $\operatorname{HFR}(\%)$ & $\mathrm{TP}$ \\
\hline 1 & 195.42 & 208.89 & 1.40 & 487.06 & 50 & 40 & 755.03 \\
\hline 2 & 198.01 & 366.00 & 1.08 & 647.86 & 46 & 41 & 1341.68 \\
\hline 3 & 719.37 & 210.01 & 1.62 & 1537.20 & 48 & 38 & 1135.27 \\
\hline 4 & 194.95 & 108.36 & 2.07 & 353.17 & 53 & 35 & 807.03 \\
\hline 5 & 273.25 & 177.43 & 2.13 & 541.55 & 54 & 34 & 992.23 \\
\hline 6 & 416.93 & 148.80 & 1.99 & 925.43 & 49 & 38 & 1624.33 \\
\hline 7 & 512.30 & 196.50 & 1.87 & 770.40 & 38 & 36 & 1352.64 \\
\hline 8 & 221.93 & 140.86 & 1.40 & 715.78 & 51 & 37 & 1077.25 \\
\hline \multicolumn{8}{|c|}{ With intermediate music } \\
\hline Weeks & LFP & HFP & FR (LF/HF) & VLFP & LFR $(\%)$ & HFR $(\%)$ & TP \\
\hline 1 & 328.81 & 159.66 & 2.66 & 734.63 & 56 & 33 & 1192.15 \\
\hline 2 & 276.77 & 247.24 & 2.65 & 540.99 & 53 & 33 & 1372.26 \\
\hline 3 & 557.20 & 622.00 & 1.90 & 680.92 & 49 & 36 & 1860.11 \\
\hline 4 & 358.27 & 112.05 & 2.55 & 846.83 & 53 & 34 & 1265.49 \\
\hline 5 & 411.24 & 229.96 & 2.41 & 928.05 & 56 & 32 & 1516.39 \\
\hline 6 & 821.28 & 176.21 & 2.50 & 664.52 & 59 & 31 & 1985.35 \\
\hline 7 & 353.89 & 310.14 & 1.98 & 757.03 & 53 & 34 & 1384.67 \\
\hline 8 & 522.42 & 468.33 & 1.34 & 632.21 & 50 & 38 & 2164.87 \\
\hline
\end{tabular}

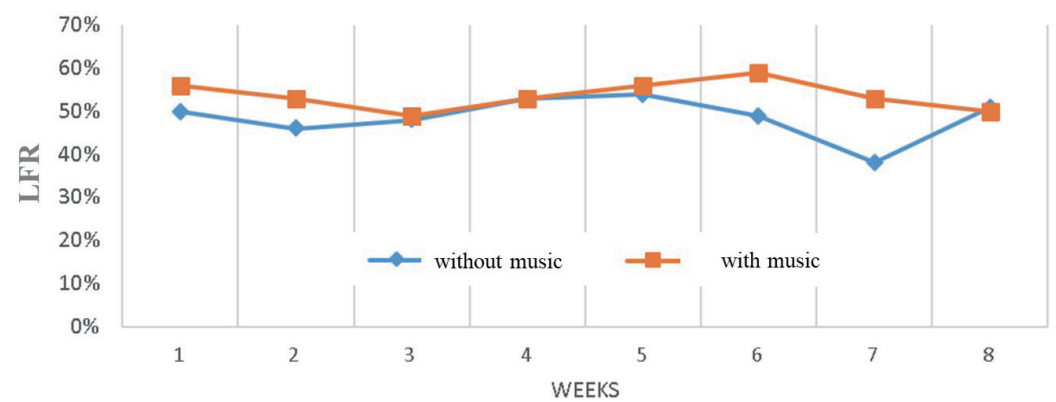

Fig. 6. (Color online) Changes in LFR without and with intermediate music in the experiment period. 


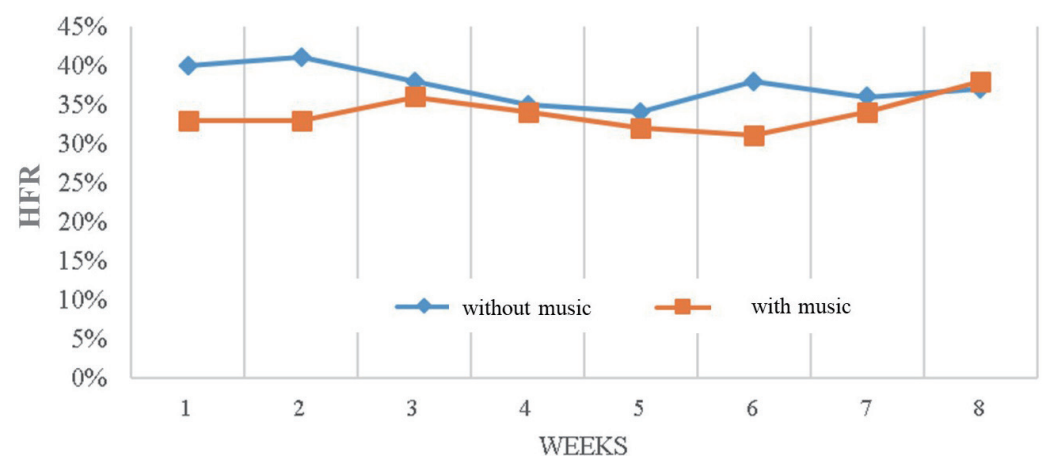

Fig. 7. (Color online) Changes in HFR without and with intermediate music in the experiment period.

\section{Slow music}

The experimental results obtained without music are shown in Table 6. Without listening to any music, HRV was measured continuously for $10 \mathrm{~min}$ in the same experiment with fast and intermediate music. LFR showed no significant difference $(F=0.13, p<0.05)$ (Fig. 8). HFR with arhythmic music was significantly higher than that without such music $(F=0.00, p<0.05)$ (Fig. 9), revealing that the parasympathetic nerve activity increased to enable notable relaxation. In the experiment without any music, TP in the second 5 min increased significantly. TP also increased slightly after eight weeks of experimentation. This means that taking a rest increased the activity of TP, thereby increasing the overall energy. The results of the experiment are shown in Table 7.

Table 6

Indicators measured with and without slow music.

\begin{tabular}{lcrcrcrr}
\hline \multicolumn{7}{c}{ Without slow music } \\
\hline Experiment Session & LFP & HFP & FR (LF/HF) & VLFP & LFR (\%) & HFR (\%) & TP \\
\hline 1 & 195.42 & 208.89 & 1.40 & 487.06 & 50 & 40 & 755.03 \\
2 & 198.01 & 366.00 & 1.08 & 647.86 & 46 & 41 & 1341.68 \\
3 & 719.37 & 210.01 & 1.62 & 1537.20 & 48 & 38 & 1135.27 \\
4 & 194.95 & 108.36 & 2.07 & 353.17 & 53 & 35 & 807.03 \\
5 & 273.25 & 177.43 & 2.13 & 541.55 & 54 & 34 & 992.23 \\
6 & 416.93 & 148.80 & 1.99 & 925.43 & 49 & 38 & 1624.33 \\
7 & 512.30 & 196.50 & 1.87 & 770.40 & 38 & 36 & 1352.64 \\
8 & 221.93 & 140.86 & 1.40 & 715.78 & 51 & 37 & 1077.25 \\
\hline & & \multicolumn{7}{c}{} & & & \\
\hline Experiment Session & LFP & HFP & FR (LF/HF) & VLFP & LFR (\%) & HFR (\%) & TP \\
\hline 1 & 326.72 & 255.44 & 1.26 & 622.90 & 46 & 40 & 1298.22 \\
2 & 345.30 & 258.38 & 1.11 & 475.00 & 45 & 43 & 1563.21 \\
3 & 1599.95 & 1588.58 & 1.02 & 1436.60 & 39 & 46 & 3577.78 \\
4 & 445.83 & 612.03 & 1.03 & 926.85 & 44 & 43 & 1984.71 \\
5 & 593.64 & 172.33 & 1.73 & 692.61 & 50 & 36 & 1200.82 \\
6 & 480.94 & 285.44 & 1.49 & 1277.92 & 45 & 42 & 2038.45 \\
7 & 991.91 & 255.47 & 1.55 & 988.00 & 51 & 38 & 1356.04 \\
8 & 351.37 & 624.76 & 1.33 & 1071.73 & 45 & 43 & 1779.40 \\
\hline
\end{tabular}




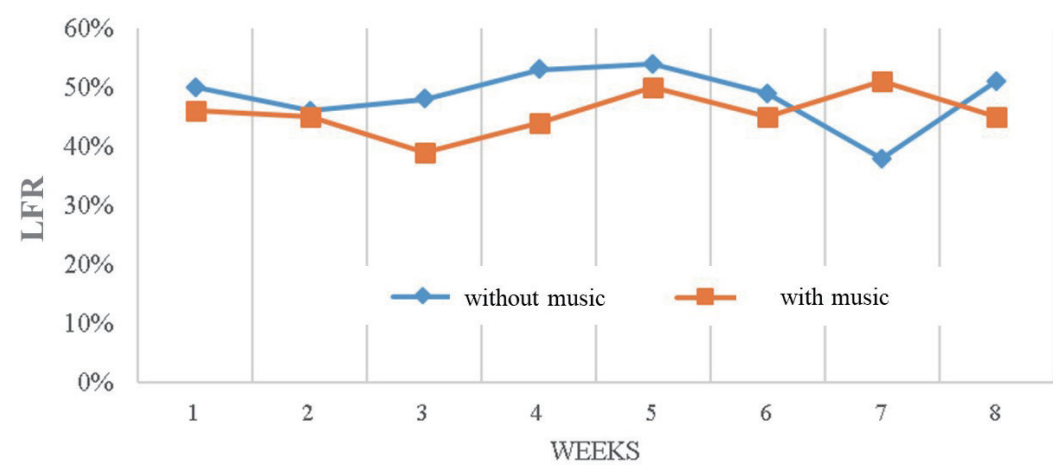

Fig. 8. (Color online) Changes in LFR with and without slow music in the experiment period.

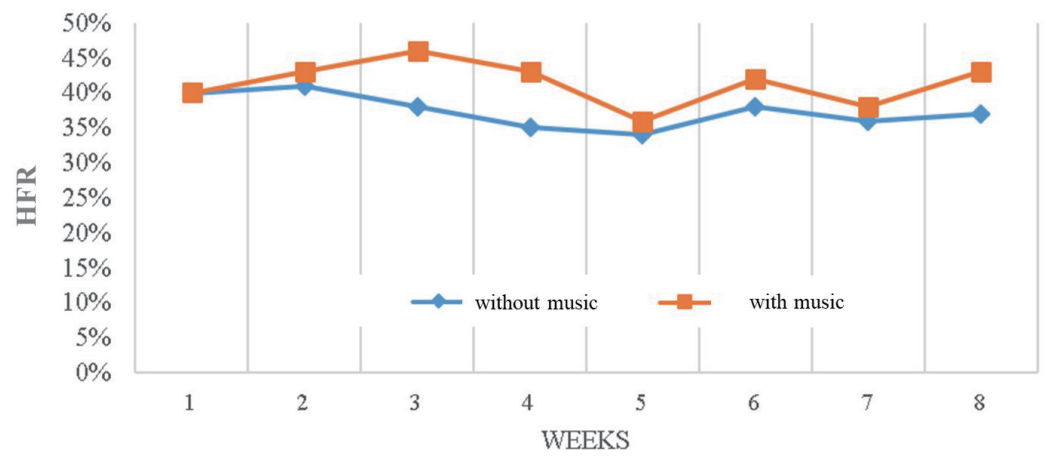

Fig. 9. (Color online) Changes in HFR with and without slow music in the experiment period.

Table 7

Results for indicators of the autonomic nervous activity obtained by t-test.

\begin{tabular}{lccc}
\hline Autonomic activity & LFR & HFR & TP \\
\hline \multirow{2}{*}{ Fast music } & Significant decrease & Significant increase & Significant increase \\
& $P=0.017$ & $P=0.005$ & $P=0.008$ \\
\hline \multirow{2}{*}{ Intermediate music } & Significant increase & Significant decrease & increase \\
& $P=0.002$ & $P=0.000$ & $P=0.230$ \\
\hline \multirow{2}{*}{ Slow music } & decrease & Significant increase & Significant increase \\
& $P=0.097$ & $P=0.007$ & $P=0.018$ \\
\hline
\end{tabular}

When the participants listened to fast music (rock music chosen by themselves), the autonomic sympathetic nerve activity was enhanced and LFR and TP increased. However, fast music decreased the parasympathetic nerve activity, which decreased HFR, resulting in inhibitory and unrelaxed states. Intermediate music (ballads and folk songs chosen by the participants) weakened the autonomic sympathetic nerve activity and increased LFR. HFR increased significantly with the enhanced parasympathetic nerve activity. However, TP did not have a significant difference. Slow music did not change the autonomic sympathetic nerve activity, but increased HFR and TP. 


\subsection{Responses of ANS}

Thinking, emotions, actions, and behaviors are controlled by the central nervous system. Response to an emotion is closely related to the $\mathrm{ANS}^{(18)}$ that regulates the muscle movement of internal organs and the production of endocrine hormones in the human body. The ANS is also responsible for the maintenance and coordination of physiological functions such as heartbeat, breathing, blood pressure, and body temperature. The ANS is divided into the sympathetic and parasympathetic nervous systems.

The autonomic nervous system in the central nervous system comprises the sympathetic nervous system and the parasympathetic nervous system. It is controlled by people's feelings, emotions, and senses. These two systems control heartbeat, breathing, gastrointestinal motility, perspiration, and sleep. The role of the sympathetic nervous system is to increase heartbeat and breathing rate when people are excited or happy, increase body temperature, sweating and blood pressure, slow down gastrointestinal peristalsis, and increase the activity of the central body system. The role of the parasympathetic nervous system is opposite to that of the sympathetic nervous system. It slows down the heartbeat and breathing, increases the rate of gastrointestinal peristalsis, and lowers blood pressure so that the activity of the primary system is reduced, and the body can rest and sleep. The interaction of the sympathetic and parasympathetic nervous systems is mutually antagonistic. In terms of its function, the sympathetic nervous system is responsible for coping with external challenges and consumes energy, whereas the parasympathetic nervous system is responsible for timely recuperation and energy conservation. The function of the parasympathetic nervous system is opposite to that of the sympathetic nervous system. The parasympathetic nervous system controls the state of relaxation, rest, and sleep of a person to save energy. When the parasympathetic nervous system is more active, the blood pressure is low and the heartbeat is slow. When the sympathetic nervous system is more active, anxiety, tightness, palpitations, and an increased blood pressure are observed. Therefore, different emotions result in different physiological responses owing to the changes in the degrees of activation of the two nervous systems in ANS. ${ }^{(7)}$ Table 8 shows the corresponding physiological and emotional responses of the ANS.

In this study, important indicators of the sympathetic and parasympathetic nervous activities in the frequency domain analysis are LFR and HFR. Fast music caused the parasympathetic nerve to increase HRF and TP, while intermediate music induced the autonomic sympathetic nerve to increase LRF and TP. Slow music increased TP but did not significantly change HRF or LFR. This implies that music affected the ANS and increased the overall energy of the human body. The rhythm, represented by BPM in this study, affected the change in emotion.

Table 8

Physiological and emotional responses of the ANS.

\begin{tabular}{lcc}
\hline Response & Sympathetic nervous activity & Parasympathetic nervous activity \\
\hline Emotional & nervousness, excitement, fear, stress & calmness, relaxation, pleasure \\
\hline Physiological & $\begin{array}{c}\text { fast heartbeat, high blood pressure, fast } \\
\text { breathing, reduced bowel movement, tight } \\
\text { muscles }\end{array}$ & $\begin{array}{c}\text { slow heartbeat, decreased blood pressure, slow } \\
\text { breathing, increased gastrointestinal motility, } \\
\text { muscle relaxation }\end{array}$ \\
\hline
\end{tabular}




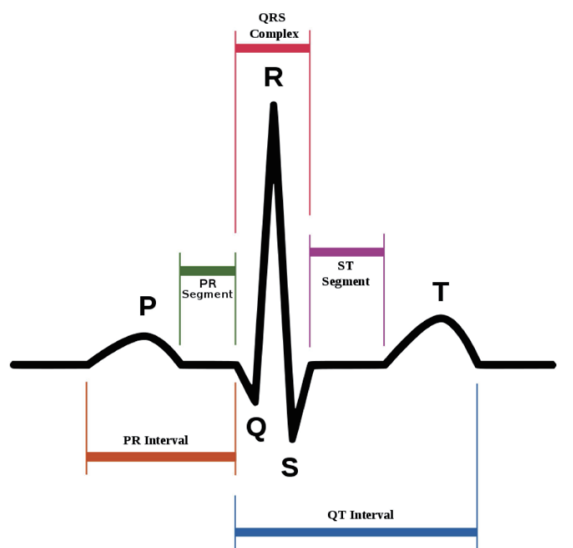

(a)

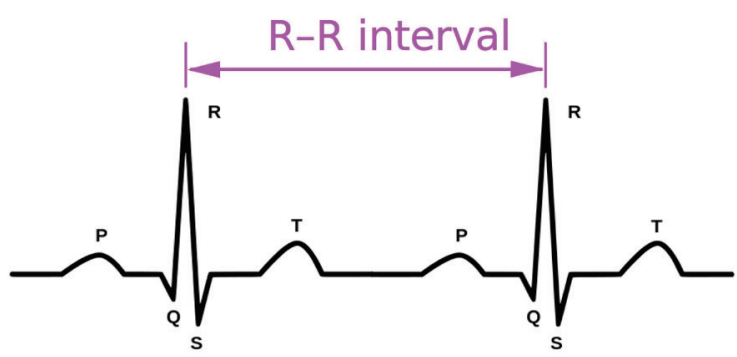

(b)

Fig. 10. (Color online) (a) Heartbeat cycle waveform ${ }^{(15)}$ and (b) R-R interval. ${ }^{(19)}$

Table 9

Effects of music on mood and heart rate.

\begin{tabular}{lcc}
\hline \multirow{2}{*}{ Music characteristics } & \multicolumn{2}{c}{ Physiological response } \\
\cline { 2 - 3 } & Heart rate & Emotional state \\
\hline Quiet and soothing & Drop & Happy, calm, sad \\
\hline Stimulating and encouraging & Rise & Excited, high, happy \\
\hline
\end{tabular}

\subsection{HR and HRV}

The frequency domain analysis is based on the HRV used to monitor and measure the physiological and emotional responses from an electrocardiogram (ECG). ECG has five waveforms, namely, P, Q, R, S, and T, among which Q, R, and S waves are usually integrated to form QRS complex waves, as shown in Fig. 10(a). As R waves are the most apparent, the distance between $\mathrm{R}$ waves is called the R-R interval, as shown in Fig. 10(b); it represents the heart rate and is an essential physiological indicator in the time domain analysis. The results of studies on the relationship among music, HR, and human emotion are summarized in Table $9 .{ }^{(19,20)}$ Fast music is considered to increase the heart rate and induce excitement, while slow music has the opposite effect. However, in this study, fast and slow music increased HFR, which is related to the enhancement of the parasympathetic nervous activity. This result suggests that the high stress level of participants was alleviated by listening to fast and slow music. The increased LFR when listening to intermediate music is considered to be related to the preference of music by the participants.

\section{Conclusions}

To understand the effect of the rhythm (tempo) of music on people's emotion, HRV was collected and analyzed with and without listening to music of different BPM ranges: fast (120-140 BPM), intermediate (60-80 BPM), and slow (less than 40 BPM). 
Experimental results showed that listening to music changed TP (an indicator of overall energy), LFR (an indicator of the sympathetic nerve activity), and HFR (an indicator of the parasympathetic nerve activity). Listening to fast music increased LFR and TP, but decreased HFR, whereas intermediate music had the opposite result. Listening to slow music increased HFR and TP, which is a mixture of the effects of fast and intermediate music.

Fast music affected the autonomic sympathetic nerve activity, resulting in increased excitability and activity, which in turn led to increased TP. The activity level of HFR of the parasympathetic nerve increased significantly with fast music, inducing an inhibitory, but not a relaxed, state. Intermediate music weakened the sympathetic nerve activity and decreased LFR, inducing a state of excitement. In contrast, HFR and TP increased significantly and the parasympathetic nerve activity was enhanced, which reflected a relaxing effect. Slow music decreased the sympathetic nerve activity, but did not significantly change LFR. Instead, HFR and TP increased, which reflected a relaxing effect.

The results of this study proved that music affects people's emotion mainly through rhythm and tempo, and suggested that the appropriate use of music may help people enhance their work performance and maintain emotional stability.

\section{Acknowledgments}

This study was supported by the Big Data Technology Research Center of Guangdong Smart Vocational Education under grant No. 3001/180113491 and Panyu Polytechnic Innovation Team under grant No. 2020CXTD003.

\section{References}

1 L. McCorry: Am. J. Pharm. 71 (2007) 78. https://doi.org/10.5688/aj710478.

2 L. Politano, A. Palladino, G. Nigro, M. Scutifero, and V. Cozza: Acta Myol. 27 (2008) 114. https://www. researchgate.net/publication/26244302_Usefulness_of heart rate_variability_as_a_predictor_of_sudden cardiac death_in_muscular_dystrophies

3 P. Palatini and S. Julius. Curr. Hypertens. Rep. 11 (2009) 199. https://doi.org/10.1007/s11906-009-0035-4 .

4 C. M. van Ravenswaaij-Arts, L. A. Kollee, J. C. Hopman, G. B. Stoelinga, and H. P. van Geijn: Ann. Intern. Med. 118 (1993) 436. https://doi.org/10.7326/0003-4819-118-6-199303150-00008

5 W. Wieling: Heart 57 (1987) 348. https://oi.org/10.1136/hrt.57.1.109-a

6 S. D. Kreibig: Bio. Psychol. 84 (2010) 394. https://doi.org/10.1016/j.biopsycho.2010.03.010

7 R. J. Davidson, K. R. Sherer, and H. H. Goldsmith: Handbook of Affective Sciences (Oxford University Press, Oxford, 2009) Chap. 45.

8 R. Hiraga, N. Kato, and N. Matsuda: Proc. 2008 IEEE Int. Conf. Systems Man and Cybernetics (IEEE, 2008) 131-136. https://doi.org/10.1109/ICSMC.2008.4811263

9 G. A. Tsihrintzis, M. Virvou, E. Alepis, and I.-O. Stathopoulou: Proc. 2008 5th Int. Conf. Information Technology (IEEE, 2008) 32-37. https://doi.org/10.1109/ITNG.2008.152

10 D. Sabatinelli, P. J. Lang, A. Keil, and M. M. Bradley: Cereb. Cortex. 17 (2007) 1085. https://doi.org/10.1093/ cercor/bhl017

11 G. Pocock, C. D. Richards, and D. A. Richards: Human Physiology (Oxford University Press, Oxford, 2013) Chap. 1.

12 U. R. Acharya, K. P. Joseph, N. Kannathal, C. M. Lim, and J. S. Suri: Med. Biol. Eng. Comput. 44 (2006) 1031. https://doi.org/10.1007/s11517-006-0119-0

13 W. Liang, J. Yuan, D. Sun, and M. Lin: JCIEE 25 (2008) 457. https://doi.org/10.1080/10170660809509108

14 Z. Li, K. Jiao, M. Chen, and C. Wang: Eur. J. Appl. Physiol. 88 (2003) 404. https://doi.org/10.1007/s00421-002$\underline{0747-5 .}$. 
15 Y. Lin: The Influences of Physical Activity Amounts to Heart Rate Variability in Different Body Weight Groups among Junior High School Pupils, Master Thesis (National Yang-Ming University, 2007).

16 M. Malik, A. J. Camm, J. T. Bigger, G. Breithardt, S. Cerutti, R. J. Cohen, P. Coumel, E. L. Fallen, H. L. Kennedy, R. E. Kleiger, F. Lombardi, A. Malliani, A. J. Moss, J. N. Rottman, G. Schmidt, P. J. Schwartz, and D. H. Singer: Eur. Heart J. 17 (1996) 354. https://pubmed.ncbi.nlm.nih.gov/8598068/

17 J. L. Jameson, A. S. Fauci, D. L. Kasper, S. L. Hauser, D. L. Longo, and J. Loscalzo: Harrison's Principles of Internal Medicine (McGraw-Hill Education, New York, 2018) Chap. 1.

18 B. Cheng and G. Liu: Proc. 2nd Int. Conf. Bioinformatics and Biomedical Engineering (IEEE, 2008) 13631366. https://doi.org/10.1109/ICBBE.2008.670

19 J. Kim and E. Andre: IEEE Trans. Pattern Anal. Mach. Intell. 30 (2008) 2067. https://doi.org/10.1109/ TPAMI.2008.26

20 P. Bason and B. Celler: Nature 238 (1972) 5362. https://doi.org/10.1038/238279a0

\section{About the Authors}

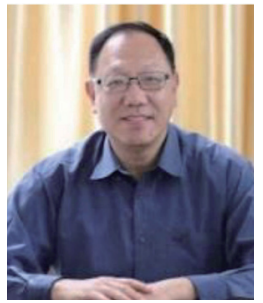

Ting-Cheng Chang received his M.S. degree from the University of Houston in 1992 and his Ph.D. degree from the University of Texas in 1996. He majored in process control and mechanical engineering. He is a professor of the Faculty of Information Engineering at Guangzhou Panyu Polytechnic, Guangdong, China. His research interests are in the field of Internet of Things, data mining, big data, and AI.

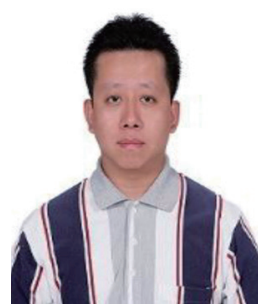

Min-Hao Wu received his Ph.D. degree from National Central University, Taoyuan, Taiwan, in 2016. He is now an associate professor of the Faculty of Information Engineering, Guangzhou Panyu Polytechnic, Guangdong, China. His research interests are in the fields of system security, mobile device security, and web security. 